Marek Fila and Johannes Lankeit*

\title{
Lack of smoothing for bounded solutions of a semilinear parabolic equation
}

https://doi.org/10.1515/anona-2020-0059

Received May 6, 2019; accepted December 1, 2019.

Abstract: We study a semilinear parabolic equation that possesses global bounded weak solutions whose gradient has a singularity in the interior of the domain for all $t>0$. The singularity of these solutions is of the same type as the singularity of a stationary solution to which they converge as $t \rightarrow \infty$.

Keywords: singular gradient; semilinear parabolic equation

MSC: 35K58, 35B44

For a bounded, smooth domain $\Omega \subset \mathbb{R}^{n}, T>0$ and $A \in \mathbb{R}$, consider solutions of the problem

$$
\begin{cases}u_{t}=\Delta u+g(u, \nabla u) & \text { in } \Omega \times(0, T), \\ u=A & \text { on } \partial \Omega \times(0, T), \\ u(\cdot, 0)=u_{0} & \text { in } \bar{\Omega} .\end{cases}
$$

It is well known (see [1, Thm. VI.4.2]) that this problem has a unique classical solution for small $T>0$ provided $g \in C^{1}\left(\mathbb{R}^{n+1}\right), u_{0} \in C^{1}(\bar{\Omega})$ and $u_{0}=A$ on $\partial \Omega$. In this paper we study a particular case of problem (0.1) in a radially symmetric setting in $B_{R}:=\left\{x \in \mathbb{R}^{n}|| x \mid<R\right\}, R>0$, where $g$ is a smooth function of $u$ and $u_{r}$ but $u_{0}$ is only Hölder continuous in $\bar{B}_{R}$. In our example, the global bounded weak solution emanating from $u_{0}$ maintains the singularity of the gradient of $u_{0}$ for all $t>0$. Thus, there is no smoothing effect which one usually expects from a semilinear uniformly parabolic equation.

The equation we will be interested in is the following:

$$
u_{t}=\Delta u+u u_{r}^{3} \quad \text { in } \quad\left(B_{R} \backslash\{0\}\right) \times(0, \infty) .
$$

(Written in Cartesian coordinates, $g$ hence corresponds to $g(x, u, \nabla u)=u\left(\nabla u \frac{x}{|x|}\right)^{3}$ for $x \in B_{R} \backslash\{0\}$.)

For $n \in \mathbb{N}, n \geq 2$, the function

$$
u^{\star}(r)=-\alpha r^{\frac{1}{3}} \quad \text { for } \quad r>0, \quad \text { where } \alpha:=\sqrt[3]{9 n-15},
$$

forms a stationary solution of (0.2) (for any $R>0$ both in $B_{R} \backslash\{0\}$, cf. Lemma 5, and - in the weak sense - in $B_{R}$, see Lemma 6).

We will impose several conditions on the initial data $u_{0}$ (and refer to (2.14) in Section 2 below for details) that, besides radial symmetry, essentially require that $u_{0}$ lies below the stationary solution, but is 'close' to it in a suitable sense. Under these conditions we will be able to show the global existence of solutions that retain the singularity in their gradient throughout the evolution.

Theorem 1. Let $n \geq 2$ and $0<R<\sqrt{\frac{3}{8}(3 n-5)(2 n-3)^{3}}$. Assume that $u_{0}$ satisfies (2.14). Then there is $a$ function

$$
u \in C\left(\bar{B}_{R} \times[0, \infty)\right) \cap C^{2,1}\left(\left(B_{R} \backslash\{0\}\right) \times(0, \infty)\right)
$$

\footnotetext{
Marek Fila, Department of Applied Mathematics and Statistics, Comenius University, Mlynská dolina, 84248 Bratislava, Slovakia, E-mail: fila@fmph.uniba.sk

*Corresponding Author: Johannes Lankeit, Department of Applied Mathematics and Statistics, Comenius University, Mlynská dolina, 84248 Bratislava, Slovakia, E-mail: jlankeit@math.upb.de
} 
which solves

$$
\begin{cases}u_{t}=\Delta u+u u_{r}^{3} & \text { in }\left(B_{R} \backslash\{0\}\right) \times(0, \infty), \\ u(0, t)=0, \quad u(R, t)=u^{\star}(R) & \text { for all } t>0, \\ u(\cdot, 0)=u_{0} & \text { in } \bar{B}_{R},\end{cases}
$$

in the classical sense. This solution is unique in the class of functions satisfying (0.4) and with $u_{r}$ being bounded from above on $\left(B_{R} \backslash\{0\}\right) \times(0, \infty)$. Moreover, it holds that

$$
\lim _{r \searrow 0} u_{r}(r, t)=-\infty \quad \text { for every } t>0 .
$$

For a more precise description of the singularity see Remark 7. Next we show that the function $u$ from Theorem 1 solves the equation from $(0.2)$ also in $B_{R} \times(0, \infty)$ in a suitable weak sense.

Theorem 2. In addition to the assumptions of Theorem 1 let $n \geq 3$. Then the solution $u$ from Theorem 1 is a weak solution of

$$
\begin{cases}u_{t}=\Delta u+u u_{r}^{3} & \text { in } B_{R} \times(0, \infty) \\ u=u^{\star}(R) & \text { on } \partial B_{R} \times(0, \infty) \\ u(\cdot, 0)=u_{0} & \text { in } \bar{B}_{R}\end{cases}
$$

By this we mean that

$$
u u_{r}^{3} \in L_{l o c}^{1}\left(B_{R} \times[0, \infty)\right) \quad \text { and } \quad \nabla u \in L_{l o c}^{1}\left(B_{R} \times[0, \infty)\right),
$$

and for every $\varphi \in C_{c}^{\infty}\left(B_{R} \times(0, \infty)\right)$ we have

$$
-\int_{0}^{\infty} \int_{B_{R}} \varphi_{t} u=-\int_{0}^{\infty} \int_{B_{R}} \nabla u \cdot \nabla \varphi+\int_{0}^{\infty} \int_{B_{R}} u u_{r}^{3} \varphi .
$$

We note that Theorem 1 guarantees that the initial and boundary conditions are satisfied.

Concerning the large-time behavior we establish the following:

Theorem 3. Under the assumptions of Theorem 1,

$$
u(\cdot, t) \rightarrow u^{\star} \text { as } t \rightarrow \infty .
$$

This convergence is uniform in $B_{R}$ and occurs with an exponential rate.

An equation closely related to (0.2) has been studied before in [2, 3], see also [4]. It was shown in [2] that interior gradient blow-up may occur for solutions of the problem

$$
\left\{\begin{array}{l}
u_{t}=u_{x x}+f(u)\left|u_{x}\right|^{m-1} u_{x}, \quad x \in(-1,1), \\
u( \pm 1, t)=A_{ \pm}
\end{array}\right.
$$

where $m>2$ and $f(u)=u$, for example. A global continuation after the interior gradient blow-up has been constructed recently in [3] for $m=3$.

For various parabolic equations, solutions with a standing or moving singularity have been investigated by many authors. We shall give some references below. But in these references it is the solution itself that is unbounded while in the present work only the gradient stays unbounded.

For the equation

$$
u_{t}=\nabla \cdot\left(u^{m-1} \nabla u\right)
$$

solutions with standing singularities were considered in [5-10] for various ranges of $m>0, m \neq 1$, and some results on moving singularities for the same equation can be found in [11]. 
Results on moving singularities for the heat equation were established in $[12,13]$ and for semilinear equations of the form

$$
u_{t}=\Delta u \pm u^{p}, \quad p>1,
$$

in [14-19]. The behaviour of solutions with standing singularity for equation (0.11) with positive sign has been studied in [20, 21].

Counterexamples to the regularizing effect of $(0.10)$ can be found in [22]. There, again, it is the solution itself that is unbounded.

Next we describe the plan of the paper. Due to the gradient singularity that the solutions have at the spatial origin, the notion of classical solvability is restricted to $\left(B_{R} \backslash\{0\}\right) \times(0, \infty)$. In Section 1 we therefore begin by establishing a connection between classical solutions in $\left(B_{R} \backslash\{0\}\right) \times(0, \infty)$ and weak solutions in $B_{R} \times(0, \infty)$.

Section 2 will be concerned with the stationary solution $u^{\star}$ mentioned in (0.3) (and already use the result of Section 1). At the end of this section, we give a precise formulation of the conditions on $u_{0}$ that the theorems require (and that involve the stationary solution).

We will construct the solutions between a super- and a subsolution. As a supersolution we will use $u^{*}$, finding the subsolution will be the goal of Section 3. To this aim, we will find a solution $v$ to a (formal) linearization of (0.5) (see Lemma 8) and then ensure that $u^{\star}-v$ is a subsolution (Lemma 10). (This is also the source of the restriction on $R$ in the theorems.)

The actual construction of solutions takes place in Section 4. We first restrict the spatial domain to $\Omega_{\varepsilon}:=$ $B_{R} \backslash B_{\varepsilon}$, for the choice of the boundary value on the new boundary $\partial B_{\varepsilon} \times(0, \infty)$ already relying on $u^{\star}-v$ from Section 3. In Section 4.1, we take care of the solvability of this problem. (Classical existence results become applicable after replacing the nonlinearity $u_{r}^{3}$ by $f\left(u_{r}\right)$, see Lemma 18 , and until Lemma 26 , we will have derived sufficient estimates allowing for removal of $f$, though still $\varepsilon$-dependent.) Section 4.2 will then be concerned with $\varepsilon$-independent estimates in preparation of a compactness argument leading to the existence of solutions. The key to this part will lie in a comparison principle applied to high powers of $u_{r}$ (see Lemma 27). This is a modification of a classical technique which involves $|\nabla u|^{2}$ and originated in [23]. Section 4.3 will contain the passage to the limit $\varepsilon>0$ (Lemma 32) and deal with (0.5) and (0.7).

In Section 5, finally, we give the proofs of the theorems. By this time, they will only consist in collecting the right lemmata previously proven, and will be accordingly short.

\section{Relation between classical and weak solutions}

Of course, every classical solution of (0.5) is also a weak solution of $(0.5)-$ in $\left(B_{R} \backslash\{0\}\right) \times(0, \infty)$, which means that the singularity appears on the boundary of the domain. In order to interpret classical solutions in $\left(B_{R} \backslash\{0\}\right) \times(0, \infty)$ as weak solutions in $B_{R} \times(0, \infty)$, we merely require suitable integrability properties of the derivative near 0 :

Lemma 4. Let $n \geq 1$ and $R>0$. Assume that a radially symmetric function

$$
u \in C\left(\bar{B}_{R} \times[0, \infty)\right) \cap C^{2,1}\left(\left(B_{R} \backslash\{0\}\right) \times(0, \infty)\right)
$$

satisfies (0.8), (0.5), and for every $T>0$ we have that

$$
\lim _{\varepsilon \rightarrow 0} \frac{1}{\varepsilon} \int_{0}^{T} \int_{0}^{\varepsilon} r^{n-1}\left|u_{r}(r, t)\right| d r d t=0 .
$$

Then (0.9) holds for every $\varphi \in C_{c}^{\infty}\left(B_{R} \times(0, \infty)\right)$. 
Proof. For every $\psi \in C_{c}^{\infty}\left(\left(B_{R} \backslash\{0\}\right) \times(0, \infty)\right)$ we obtain

$$
-\int_{0}^{\infty} \int_{B_{R}} \psi_{t} u=-\int_{0}^{\infty} \int_{B_{R}} \nabla u \cdot \nabla \psi+\int_{0}^{\infty} \int_{B_{R}} u u_{r}^{3} \psi,
$$

as $u$ solves the equation classically in $\left(B_{R} \backslash\{0\}\right) \times(0, \infty)$.

We introduce a non-decreasing cut-off function $\chi \in C^{\infty}(\mathbb{R})$ with $0 \leq \chi^{\prime} \leq 2$ and $\chi(0)=0, \chi \equiv 1$ on $[1, \infty)$ and let $\chi_{\varepsilon}(x):=\chi\left(\frac{|x|}{\varepsilon}\right)$.

We let $\varphi \in C_{c}^{\infty}\left(B_{R} \times(0, \infty)\right)$ and note that for every positive $\varepsilon, \psi:=\chi_{\varepsilon} \varphi$ belongs to $C_{c}^{\infty}\left(\left(B_{R} \backslash\{0\}\right) \times(0, \infty)\right)$.

$$
\begin{aligned}
-\int_{0}^{\infty} \int_{B_{R}} \varphi_{t} u= & -\int_{0}^{\infty} \int_{B_{R}} \psi_{t} u-\int_{0}^{\infty} \int_{B_{R}} \varphi_{t}\left(1-\chi_{\varepsilon}\right) u \\
= & -\int_{0}^{\infty} \int_{B_{R}} \nabla u \cdot \nabla \psi+\int_{0}^{\infty} \int_{B_{R}} u u_{r}^{3} \psi-\int_{0}^{\infty} \int_{B_{R}} \varphi_{t}\left(1-\chi_{\varepsilon}\right) u \\
= & -\int_{0}^{\infty} \int_{B_{R}} \chi_{\varepsilon} \nabla u \cdot \nabla \varphi-\int_{0}^{\infty} \int_{B_{R}} \varphi \nabla u \cdot \nabla \chi_{\varepsilon} \\
& +\int_{0}^{\infty} \int_{B_{R}} u u_{r}^{3} \varphi \chi_{\varepsilon}-\int_{0}^{\infty} \int_{B_{R}} \varphi_{t}\left(1-\chi_{\varepsilon}\right) u
\end{aligned}
$$

for every $\varepsilon>0$. As $\chi_{\varepsilon} \rightarrow 1$ a.e. in $\operatorname{supp} \varphi$ and by (0.8) and boundedness of $u$ each of the functions $\nabla u \cdot \nabla \varphi$, $u u_{r}^{3} \varphi, \varphi_{t} u$ belongs to $L^{1}(\operatorname{supp} \varphi)$,

$$
\begin{gathered}
-\int_{0}^{\infty} \int_{B_{R}} \chi_{\varepsilon} \nabla u \cdot \nabla \varphi+\int_{0}^{\infty} \int_{B_{R}} u u_{r}^{3} \varphi \chi_{\varepsilon}-\int_{0}^{\infty} \int_{B_{R}} \varphi_{t}\left(1-\chi_{\varepsilon}\right) u \\
\rightarrow-\int_{0}^{\infty} \int_{B_{R}}^{\infty} \nabla u \cdot \nabla \varphi+\int_{0}^{\infty} \int_{B_{R}} u u_{r}^{3} \varphi \quad \text { as } \varepsilon \rightarrow 0
\end{gathered}
$$

by Lebesgue's dominated convergence theorem.

Moreover, $\left|\nabla \chi_{\varepsilon}(x)\right|=\left|\chi_{\varepsilon r}(r)\right|=\frac{1}{\varepsilon} \chi^{\prime}\left(\frac{r}{\varepsilon}\right) \leq \frac{2}{\varepsilon}$ if $r=|x|<\varepsilon$ and $\left|\nabla \chi_{\varepsilon}(x)\right|=0$ if $|x|>\varepsilon$. With $T>0$ such that $\operatorname{supp} \varphi \subset B_{R} \times(0, T)$, we have

$$
\left|\int_{0}^{\infty} \int_{B_{R}} \varphi \nabla u \cdot \nabla \chi_{\varepsilon}\right| \leq\|\varphi\|_{\infty} \int_{0}^{T} \int_{B_{R}}|\nabla u|\left|\nabla \chi_{\varepsilon}\right| \leq \frac{2}{\varepsilon}\|\varphi\|_{\infty} \int_{0}^{T} \int_{0}^{\varepsilon} r^{n-1}\left|u_{r}(r, t)\right| d r d t,
$$

which vanishes as $\varepsilon \rightarrow 0$ according to (1.12), and (0.9) follows.

\section{The stationary solution and conditions on the initial data}

In (0.3), we have introduced a stationary solution $u^{*}$ to $(0.2)$. In this section we first prove that the function from (0.3) actually has this property (see Lemma 5 for the classical, Lemma 6 for the weak sense) and then formulate the conditions on the initial data, which involve relations with $u^{\star}$ and whose formulation we therefore had postponed.

Lemma 5. Let $n \geq 2$. Then the function $u^{\star}$ from (0.3) solves

$$
\Delta u^{\star}+u^{\star}\left(u_{r}^{\star}\right)^{3}=0 \quad \text { in } \mathbb{R}^{n} \backslash\{0\} .
$$


Proof. We use radial symmetry and the explicit form of $u^{\star}$ to write

$$
\begin{aligned}
\Delta u^{\star}+u^{\star}\left(u_{r}^{\star}\right)^{3} & =r^{1-n}\left(r^{n-1}\left(-\frac{\alpha}{3} r^{-\frac{2}{3}}\right)\right)_{r}+\alpha r^{\frac{1}{3}}\left(\frac{\alpha}{3} r^{-\frac{2}{3}}\right)^{3} \\
& =\frac{\alpha}{27} r^{-\frac{5}{3}}\left(15-9 n+\alpha^{3}\right)=0 .
\end{aligned}
$$

Lemma 6. Let $n \geq 2$. Then for any $R>0$ the function $u^{\star}$ defined in (0.3) is a weak solution of (0.7).

Proof. In order to apply Lemma 4, we only have to check integrability of $u^{\star}(r)\left(u_{r}^{\star}\right)^{3}(r)=\frac{\alpha^{4}}{27} r^{\frac{1}{3}-2}$ and $u_{r}^{\star}(r)=$ $\frac{\alpha}{3} r^{-\frac{2}{3}}$, which is satisfied, and

$$
\lim _{\varepsilon \rightarrow 0} \frac{1}{\varepsilon} \int_{0}^{\varepsilon} r^{n-1}\left|u_{r}^{\star}(r)\right| d r=\lim _{\varepsilon \rightarrow 0} \frac{\alpha}{3 n-2} \varepsilon^{n-\frac{5}{3}}=0 .
$$

Now and in the following, given any $n \in \mathbb{N}$ we let

$$
v:=v(n):=\frac{1}{6} \sqrt{36 n^{2}-96 n+61}
$$

Having introduced $u^{\star}$ and $v$, we are now in a position to give the conditions on initial data that Theorems 1,2 and 3 have posed.

$$
\begin{aligned}
& u_{0} \in C^{2}\left(\bar{B}_{R} \backslash\{0\}\right), \\
& u_{0} \text { is radially symmetric, } \\
& u^{\star} \geq u_{0}, \\
& \underset{r \searrow 0}{\limsup }\left|r^{\frac{3}{2}-n-v}\left(u^{\star}(r)-u_{0}(r)\right)\right|<\infty, \\
& u_{0}(R)=u^{\star}(R), \\
& \text { there is } C>0 \text { such that } 0 \geq u_{0 r}(r) \geq-C r^{-\frac{2}{3}} \quad \text { for every } r \in(0, R) .
\end{aligned}
$$

Remark 7. The shape of the solution from Theorem 1 near the singularity of its gradient can be described more precisely than in ( 0.6 ) by saying that (2.14d) continues to hold for $t>0$ in the sense that

$$
\underset{r \searrow 0}{\limsup }\left|r^{\frac{3}{2}-n-v}\left(u^{\star}(r)-u(r, t)\right)\right|<\infty \quad \text { for all } t>0 .
$$

We will include a proof in the proof of Theorem 1 in Section 5.

\section{Finding a subsolution}

In order to construct a subsolution of (0.5) near $u^{\star}$, we first find a solution of the (formal) linearization of (0.5) around $u^{*}$.

Lemma 8. Let $n \geq 2, C>0, v$ as in (2.13), $\lambda>0$. Then the function

$$
v(r, t):=C e^{-\lambda^{2} t} r^{n-\frac{3}{2}} J_{v}(\lambda r), \quad r>0, t>0,
$$

where $J_{v}$ denotes the Bessel function of the first kind of order $v$, solves

$$
v_{t}=\Delta v+3 u^{\star} u_{r}^{\star 2} v_{r}+u_{r}^{\star 3} v \quad \text { in }\left(\mathbb{R}^{n} \backslash\{0\}\right) \times(0, \infty)
$$

with $u^{\star}$ taken from (0.3). 
Proof. Let us recall that the function defined by $\chi(r):=J_{v}(\lambda r), r>0$, satisfies

$$
r^{2} \chi^{\prime \prime}(r)+r \chi^{\prime}(r)+\left(\lambda^{2} r^{2}-v^{2}\right) \chi=0 \quad \text { for every } r>0 .
$$

We abbreviate $A:=4-2 n$ and $B:=\frac{3 n-5}{9}$ and $\delta:=n-\frac{3}{2}$ and note that

$$
2 \delta+A=1
$$

and

$$
\delta(\delta-1)+A \delta-B=-n^{2}+\frac{8 n}{3}-\frac{61}{36}=-v^{2},
$$

so that (3.16), (3.17) and (3.18) for $\psi(r):=r^{\delta} \chi(r), r>0$, entail

$$
\begin{aligned}
r^{2} \psi^{\prime \prime}(r) & +A r \psi^{\prime}(r)+\lambda^{2} r^{2} \psi(r)-B \psi(r)=r^{2}\left(r^{\delta} \chi\right)^{\prime \prime}+A r\left(r^{\delta} \chi\right)^{\prime}-B r^{\delta} \chi \\
& =r^{2} \delta(\delta-1) r^{\delta-2} \chi+2 r^{2} \delta r^{\delta-1} \chi^{\prime}+r^{2} r^{\delta} \chi^{\prime \prime}+A \delta r^{\delta} \chi+A r^{\delta+1} \chi^{\prime}-B r^{\delta} \chi \\
& =r^{\delta}\left(r^{2} \chi^{\prime \prime}+(2 \delta+A) r \chi^{\prime}+(\delta(\delta-1)+A \delta-B) \chi\right) \\
& =r^{\delta}\left(r^{2} \chi^{\prime \prime}+r \chi^{\prime}-v^{2} \chi\right)=-r^{\delta} r^{2} \lambda^{2} \chi=-r^{2} \lambda^{2} \psi \quad \text { for } r>0,
\end{aligned}
$$

and

$$
v(r, t)=C e^{-\lambda^{2} t} r^{n-\frac{3}{2}} J_{v}(\lambda r)=C e^{-\lambda^{2} t} \psi(r), \quad r>0, t>0,
$$

solves

$$
\begin{aligned}
v_{t} & =C e^{-\lambda^{2} t}\left(-\lambda^{2} \psi\right)=C e^{-\lambda^{2} t}\left(\psi^{\prime \prime}+\frac{A}{r} \psi^{\prime}-\frac{B}{r^{2}} \psi\right) \\
& =v_{r r}+\frac{4-2 n}{r} v_{r}+\frac{5-3 n}{9 r^{2}} v=v_{r r}+\frac{n-1}{r} v_{r}-\frac{\alpha^{3}}{3 r} v_{r}-\frac{\alpha^{3}}{27 r^{2}} v \\
& =\Delta v+3 u^{\star} u_{r}^{\star 2} v_{r}+u_{r}^{\star 3} v \quad \text { in }\left(\mathbb{R}^{n} \backslash\{0\}\right) \times(0, \infty),
\end{aligned}
$$

where we have used that $\alpha=\sqrt[3]{9 n-15}$ and $u^{\star}(r)=-\alpha r^{\frac{1}{3}}$.

Definition 9. With $v$ from (2.13), we let $x_{0}>0$ and $x_{1} \in\left(0, x_{0}\right)$ be the first positive roots of the Bessel function $J_{v}$ of the first kind and its derivative $J_{v}^{\prime}$, respectively. (As $v>0, J_{v}$ and $J_{v}^{\prime}$ are positive on $\left(0, x_{0}\right)$ and $\left(0, x_{1}\right)$, respectively.)

Lemma 10. Let $n \geq 2, C>0, \lambda>0$ and, with $x_{1}$ from Definition 9,

$$
0<R<\min \left\{\frac{x_{1}}{\lambda}, \sqrt{\frac{3}{8}(3 n-5)(2 n-3)^{3}}\right\} .
$$

With $v$ from (3.15), the function

$$
u:=u^{\star}-v
$$

then satisfies

$$
u_{t} \leq \Delta u+u u_{r}^{3} \quad \text { in }\left(B_{R} \backslash\{0\}\right) \times(0, \infty) .
$$

Proof. For $u=u^{\star}-v$, we have

$$
\begin{aligned}
-u u_{r}^{3}= & -\left(u^{\star}-v\right)\left(u^{*}-v\right)_{r}^{3}=-u^{\star}\left(u^{*}-v\right)_{r}^{3}+v\left(u^{\star}-v\right)_{r}^{3} \\
= & -u^{\star}\left(u_{r}^{\star}\right)^{3}+3 u^{\star}\left(u_{r}^{\star}\right)^{2} v_{r}-3 u^{\star} u_{r}^{\star} v_{r}^{2}+u^{\star} v_{r}^{3} \\
& +\left(u_{r}^{*}\right)^{3} v-3\left(u_{r}^{\star}\right)^{2} v v_{r}+3 u_{r}^{\star} v v_{r}^{2}-v v_{r}^{3} \quad \text { in }\left(B_{R} \backslash\{0\}\right) \times(0, \infty) .
\end{aligned}
$$

As $u^{\star}$ is a stationary solution according to Lemma 5 and by Lemma $8 v$ solves the linearized equation, we conclude

$$
u_{t}^{\star}-\Delta u^{\star}-u^{\star}\left(u_{r}^{\star}\right)^{3}=0 \quad \text { in }\left(B_{R} \backslash\{0\}\right) \times(0, \infty)
$$


and

$$
-v_{t}+\Delta v+3 u^{*}\left(u_{r}^{*}\right)^{2} v_{r}+\left(u_{r}^{*}\right)^{3} v=0 \quad \text { in }\left(B_{R} \backslash\{0\}\right) \times(0, \infty) .
$$

Accordingly, in $\left(B_{R} \backslash\{0\}\right) \times(0, \infty)$ we obtain

$$
\begin{aligned}
u_{t}-\Delta u-u u_{r}^{3}= & u_{t}^{\star}-v_{t}-\Delta u^{*}+\Delta v-u^{\star}\left(u_{r}^{*}\right)^{3}+3 u^{\star}\left(u_{r}^{*}\right)^{2} v_{r}-3 u^{*} u_{r}^{*} v_{r}^{2}+u^{\star} v_{r}^{3} \\
& +\left(u_{r}^{\star}\right)^{3} v-3\left(u_{r}^{\star}\right)^{2} v v_{r}+3 u_{r}^{\star} v v_{r}^{2}-v v_{r}^{3} \\
= & -3 u^{*} u_{r}^{\star} v_{r}^{2}-3\left(u_{r}^{\star}\right)^{2} v v_{r}+u^{\star} v_{r}^{3}+3 u_{r}^{*} v v_{r}^{2}-v v_{r}^{3} \\
= & -\alpha^{2} C^{2} r^{-\frac{1}{3}} e^{-2 \lambda^{2} t}\left(\psi^{\prime}(r)\right)^{2}+\alpha C^{2} r^{-\frac{2}{3}} e^{-2 \lambda^{2} t} \psi(r) \psi^{\prime}(r) \\
& -\alpha C^{3} r^{\frac{1}{3}} e^{-3 \lambda^{2} t}\left(\psi^{\prime}(r)\right)^{3}-\alpha C^{3} r^{-\frac{2}{3}} e^{-3 \lambda^{2} t} \psi(r)\left(\psi^{\prime}(r)\right)^{2} \\
& -C^{4} e^{-4 \lambda^{2} t} \psi(r)\left(\psi^{\prime}(r)\right)^{3} .
\end{aligned}
$$

Due to $r \lambda \leq R \lambda \leq x_{1}=\min \left\{x_{0}, x_{1}\right\}$, we have that

$$
\psi^{\prime}(r)=\left(n-\frac{3}{2}\right) r^{n-\frac{5}{2}} J_{v}(\lambda r)+\lambda r^{n-\frac{3}{2}} J_{v}^{\prime}(\lambda r) \geq 0 \quad \text { for all } r \in(0, R),
$$

hence

$$
\begin{aligned}
u_{t}-\Delta u-u u_{r}^{3} & \leq-\alpha^{2} C^{2} r^{-\frac{1}{3}} e^{-2 \lambda^{2} t}\left(\psi^{\prime}(r)\right)^{2}+\alpha C^{2} r^{-\frac{2}{3}} e^{-2 \lambda^{2} t} \psi(r) \psi^{\prime}(r) \\
& =\alpha C^{2} e^{-2 \lambda^{2} t} \psi^{\prime}(r) r^{-\frac{2}{3}}\left(-\alpha r^{\frac{1}{3}} \psi^{\prime}(r)+\psi(r)\right) \quad \text { in }(0, R) .
\end{aligned}
$$

From (3.21) and $\lambda R<x_{1}=\min \left\{x_{0}, x_{1}\right\}$, we can also infer

$$
\frac{\psi^{\prime}(r)}{\psi(r)}=r^{-1}\left[\left(n-\frac{3}{2}\right)+\frac{r \lambda J_{v}^{\prime}(r \lambda)}{J_{v}(r \lambda)}\right] \geq\left(n-\frac{3}{2}\right) r^{-1} \quad \text { for all } r \in(0, R),
$$

so that

$$
-\alpha r^{\frac{1}{3}} \psi^{\prime}(r)+\psi(r) \leq\left(-\alpha r^{-\frac{2}{3}}\left(n-\frac{3}{2}\right)+1\right) \psi(r) \leq\left(-\alpha R^{-\frac{2}{3}}\left(n-\frac{3}{2}\right)+1\right) \psi(r) \leq 0
$$

for every $r \in(0, R)$, because $R^{-\frac{2}{3}} \geq\left(\frac{3}{8}(3 n-5)(2 n-3)^{3}\right)^{-\frac{1}{3}}=\left(\alpha\left(n-\frac{3}{2}\right)\right)^{-1}$, hence (3.22) turns into (3.20).

\section{Existence}

\subsection{An approximate problem}

Construction of the solution to (0.2) will be based on an appropriately modified problem on $\left(B_{R} \backslash B_{\varepsilon}\right) \times(0, \infty)$. In preparation of suitable initial data, we first turn our attention to $u_{0}$.

Lemma 11. Let $n \geq 2,0<R<\sqrt{\frac{3}{8}(3 n-5)(2 n-3)^{3}}$. Assume that $u_{0}$ satisfies (2.14). Let $\lambda>0$ be such that $\lambda R<x_{1}$. There is $C>0$ so that $v$ from (3.15) satisfies

$$
u_{0} \geq u^{\star}-v(\cdot, 0) \quad \text { in } B_{R} .
$$

Proof. Since $\lambda R<x_{0}$, known asymptotics of the Bessel function [24, p. 360, (9.1.7)] yields the existence of $c_{1}=c_{1}(\lambda)>0$ such that $c_{1} r^{v} \leq J_{v}(\lambda r)$ for every $r \in[0, R]$. Therefore, (2.14d) implies that for some $c_{2}>0$ we obtain

$$
\frac{\left|u_{0}(r)-u^{*}(r)\right|}{r^{n-\frac{3}{2}} J_{v}(\lambda r)} \leq c_{2} \quad \text { for every } r \in(0, R) .
$$

If we let $C \geq c_{2}$, this coincides with (4.23). 
Definition 12. Now and in all of the following, we let $n, C, R, \lambda, v$ be as in Lemma 10 and Lemma 11.

Definition 13. Let $\varepsilon>0$ and $u_{0}$ satisfy (2.14). We denote $\Omega_{\varepsilon}:=B_{R} \backslash B_{\varepsilon}$. Moreover, let $u_{0 \varepsilon} \in C^{2}\left(\overline{\Omega_{\varepsilon}}\right)$ be radially symmetric and such that

$$
\begin{aligned}
& u_{0 \varepsilon}(\varepsilon)=u^{\star}(\varepsilon)-v(\varepsilon, 0), \\
& u_{0 r} \leq u_{0 \varepsilon r} \leq 0, \\
& u^{\star} \geq u_{0 \varepsilon} \geq u^{\star}-v(\cdot, 0), \\
& u_{0 \varepsilon}=u_{0} \text { on the set }\left\{r \in(\varepsilon, R] \mid u_{0}(r)<u^{\star}(\varepsilon)-v(\varepsilon, 0)-\varepsilon\right\} .
\end{aligned}
$$

Remark 14. For (4.24c), we rely on Lemma 11; that the other conditions can be fulfilled is more immediate from (2.14).

Remark 15. As $u^{\star}(\varepsilon)-v(\varepsilon, 0)-\varepsilon \rightarrow 0$ as $\varepsilon \rightarrow 0$, (4.24d) ensures that for every $\delta>0$ there is $\varepsilon_{0}>0$ such that for all $\varepsilon \in\left(0, \varepsilon_{0}\right)$ we have $u_{0 \varepsilon}=u_{0}$ on $B_{R} \backslash B_{\delta}$.

Definition 16. Let $\varepsilon>0$. First let us note that

$$
c_{v}:=-e^{\lambda^{2} t} v(\varepsilon, t)
$$

is positive and constant with respect to $t$ according to (3.15).

We choose $c_{\varepsilon}^{\star}>1$ large enough so as to satisfy

$$
\begin{aligned}
c_{\varepsilon}^{\star} & >\sup _{[\varepsilon, R]}\left|u_{r}^{\star}\right|, \\
c_{\varepsilon}^{\star} & >\sup _{[\varepsilon, R]}\left|\left(u^{\star}-v(\cdot, 0)\right)_{r}\right|, \\
c_{\varepsilon}^{\star} & >\sup _{[\varepsilon, R]}\left|u_{0 \varepsilon r}\right|, \\
c_{V}+ & \frac{n-1}{\varepsilon} c_{\varepsilon}^{\star}+u^{\star}(\varepsilon)\left(c_{\varepsilon}^{\star}\right)^{3} \leq 0 .
\end{aligned}
$$

Definition 17. We let $f_{\varepsilon} \in C_{c}^{\infty}(\mathbb{R})$ be such that $f_{\varepsilon}(s)=s^{3}$ for every $s \in\left[-c_{\varepsilon}^{\star}, c_{\varepsilon}^{\star}\right]$ (with $c_{\varepsilon}^{\star}$ from Definition 16) and $f_{\varepsilon} \leq 0$ on $(-\infty, 0)$.

With $u_{0 \varepsilon}$ and $f_{\varepsilon}$ as in Definitions 13 and 17, we now consider

$$
\begin{cases}u_{\varepsilon t}=\Delta u_{\varepsilon}+u_{\varepsilon} f_{\varepsilon}\left(u_{\varepsilon r}\right) & \text { in } \Omega_{\varepsilon} \times(0, \infty), \\ \left.u_{\varepsilon}(\cdot, t)\right|_{\partial B_{\varepsilon}}=\left.\left(u^{\star}-v(\cdot, t)\right)\right|_{\partial B_{\varepsilon}} & \text { for all } t>0, \\ \left.u_{\varepsilon}(\cdot, t)\right|_{\partial B_{R}}=u_{0}(R)=u^{\star}(R) & \text { for all } t>0, \\ u_{\varepsilon}(\cdot, 0)=u_{0 \varepsilon} & \text { in } \overline{\Omega_{\varepsilon}}\end{cases}
$$

By classical theory for parabolic PDEs, this problem has a solution.

Lemma 18. Let $\varepsilon>0$. Then (4.26) has a unique solution

$$
u_{\varepsilon} \in C^{\beta, \frac{\beta}{2}}\left(\overline{\Omega_{\varepsilon}} \times[0, \infty)\right) \cap C^{2+\beta, 1+\frac{\beta}{2}}\left(\Omega_{\varepsilon} \times(0, \infty)\right) \quad \text { with } \quad \nabla u_{\varepsilon} \in L_{l o c}^{\infty}\left(\overline{\Omega_{\varepsilon}} \times[0, \infty)\right)
$$

for some $\beta \in(0,1)$. This solution is radially symmetric.

Proof. Boundedness of $f_{\varepsilon}$ and the regularity requirements on $u_{0 \varepsilon}$ ensure applicability of [1, Thm. V.6.2], which yields existence and uniqueness of the solution. Radial symmetry of $u_{0 \varepsilon}$ together with the uniqueness assertion implies radial symmetry of the solution. 
Later (in Lemmata 25 and 27) we want to invoke comparison principles for the derivative. In order to make them applicable, we need slightly more regularity than provided by Lemma 18.

Lemma 19. Let $\varepsilon>0$. Then there is $\beta \in(0,1)$ such that

$$
u_{\varepsilon} \in C^{3+\beta, \frac{3+\beta}{2}}\left(\Omega_{\varepsilon} \times(0, \infty)\right) \quad \text { and } \quad \nabla u_{\varepsilon} \in C^{\beta, \frac{\beta}{2}}\left(\overline{\Omega_{\varepsilon}} \times[0, \infty)\right) .
$$

Proof. Letting $\eta \in C_{c}^{\infty}\left(\Omega_{\varepsilon} \times(0, \infty)\right)$ we observe that $\eta u$ solves $(\eta u)_{t}=\Delta(\eta u)+g$, where $g=-\eta_{t} u-2 \nabla \eta \cdot \nabla u-$ $u \Delta \eta+\eta u f_{\varepsilon}\left(u_{r}\right)$ and that, thanks to $u \in C^{2+\beta, 1+\frac{\beta}{2}}(\operatorname{supp} \eta)$ by Lemma $18, g \in C^{1+\beta, \frac{1+\beta}{2}}\left(\overline{\Omega_{\varepsilon}} \times(0, \infty)\right)$. [1, Thm. IV.5.2] therefore implies $\eta u \in C^{3+\beta, \frac{3+\beta}{2}}\left(\overline{\Omega_{\varepsilon}} \times[0, \infty)\right)$. Hölder continuity of $\nabla u_{\varepsilon}$ up to $t=0$ and to the spatial boundary follows from [25, Thm. 4.6].

As a first estimate of $u_{\varepsilon}$, the following lemma not only affirms boundedness of $u_{\varepsilon}$, but also forms the foundation of estimate (4.30) for $u$.

Lemma 20. Let $\varepsilon>0$. Then

$$
u^{\star} \geq u_{\varepsilon} \geq u^{\star}-v \quad \text { in } \Omega_{\varepsilon} \times(0, \infty) \text {. }
$$

Proof. Due to (4.25a) and (4.25b), each of the functions $w \in\left\{u^{\star}, u_{\varepsilon}, u^{\star}-v\right\}$ satisfies $f_{\varepsilon}\left(w_{r}\right)=w_{r}^{3}$ in $\Omega_{\varepsilon} \times(0, \infty)$ and hence for $w \in\left\{u^{\star}, u_{\varepsilon}\right\}$ we have

$$
w_{t}=\Delta w+f_{\varepsilon}\left(w_{r}\right) w,
$$

whereas $w_{t} \leq \Delta w+f_{\varepsilon}\left(w_{r}\right) w$ for $w=u^{\star}-v$ (cf. Lemma 10). By construction, $u^{\star}(R)=u_{\varepsilon}(R, t) \geq u^{\star}(R)-v(R, t)$ and $u^{\star}(\varepsilon) \geq u_{\varepsilon}(\varepsilon, t)=u^{\star}(\varepsilon)-v(\varepsilon, t)$ for all $t>0$, and $u^{\star} \geq u_{0 \varepsilon} \geq u^{\star}-v(\cdot, 0)$, so that the comparison principle ([4, Prop. 52.6]) implies (4.27).

We prepare for an estimate of $u_{\varepsilon r}$ by comparison, first providing some information on its value on the spatial boundary, beginning with the outer part $\partial B_{R} \times(0, \infty)$.

Lemma 21. For every $\varepsilon>0$ and $t>0$ we have

$$
u_{r}^{\star}(R) \leq u_{\varepsilon r}(R, t) \leq 0
$$

Proof. Since $u^{\star}(R)=u_{\varepsilon}(R, t)$ for all $t>0$, (4.27) shows that $u_{r}^{\star}(R) \leq u_{\varepsilon r}(R, t)$ for all $t>0$. Moreover, $\underline{u}(r, t):=$ $u^{\star}(R),(r, t) \in[\varepsilon, R] \times[0, \infty)$, satisfies $\underline{u}_{t} \leq \Delta \underline{u}+f\left(\underline{u}_{r}\right) \underline{u}$ in $(\varepsilon, R) \times(0, \infty)$ and $\underline{u}(R, t) \leq u_{\varepsilon}(R, t), \underline{u}(\varepsilon, t) \leq u_{\varepsilon}(\varepsilon, t)$ for all $t>0$ and $\underline{u}(r, 0) \leq u_{\varepsilon}(r, 0)$ for all $r \in(\varepsilon, R)$. By the comparison principle [4, Prop. 52.6] therefore $u_{\varepsilon}(r, t) \geq u^{\star}(R)=u_{\varepsilon}(R, t)$ for every $(r, t) \in(0, R) \times(0, \infty)$ so that $u_{\varepsilon r}(R, t) \leq 0$ for every $t>0$.

On the inner boundary, we first establish the sign of $u_{\varepsilon r}$.

Lemma 22. For every $\varepsilon>0$ and $t>0$ it holds that

$$
u_{\varepsilon r}(\varepsilon, t) \leq 0
$$

Proof. With $\mathcal{M}[\phi]:=\phi_{t}-\Delta \phi-u_{\varepsilon} u_{\varepsilon r}^{2} \phi_{r}$ and $\bar{u}(x, t):=u^{\star}(\varepsilon)-v(\varepsilon, t)$ for $(x, t) \in \overline{\Omega_{\varepsilon}} \times[0, \infty)$, we have

$$
\mathcal{M}\left[u_{\varepsilon}\right]=0, \quad \mathcal{M}[\bar{u}]=\bar{u}_{t}=-v_{t}(\varepsilon, t) \geq 0 \quad \text { in } \Omega_{\varepsilon} \times(0, \infty),
$$

which together with $u_{\varepsilon}(\varepsilon, t)=\bar{u}(\varepsilon, t), u_{\varepsilon}(R, t)=u^{\star}(R) \leq \bar{u}(R, t)$ for all $t>0$ and the consequence $u_{0 \varepsilon}(r) \leq$ $u_{0 \varepsilon}(\varepsilon)=\bar{u}(r, 0)$ of (4.24b) and (4.24a) enables us to invoke [4, Prop. 52.6] once more to conclude $u_{\varepsilon}(r, t) \leq$ $\bar{u}(r, t)=u_{\varepsilon}(\varepsilon, t)$ for all $r \in(\varepsilon, R)$ and $t>0$, which implies $u_{\varepsilon r}(\varepsilon, t) \leq 0$ for all $t>0$.

The upper estimates in Lemma 21 and Lemma 22 determine the sign of $u_{\varepsilon r}$ throughout $\Omega_{\varepsilon} \times[0, \infty)$.

Lemma 23. Let $\varepsilon>0$. Then

$$
u_{\varepsilon r} \leq 0 \quad \text { in } \Omega_{\varepsilon} \times[0, \infty) \text {. }
$$


Proof. As $w:=u_{\varepsilon r}$ belongs to $C\left(\overline{\Omega_{\varepsilon}} \times(0, \infty)\right) \cap C\left([0, \infty) ; L^{2}\left(\overline{\Omega_{\varepsilon}}\right)\right)$ with $w_{t}, \nabla w, D^{2} w \in L_{l o c}^{2}\left(\Omega_{\varepsilon} \times(0, \infty)\right)$ by Lemma 19, solves $w_{t}=\Delta w+f_{\varepsilon}\left(u_{\varepsilon r}\right) w+u_{\varepsilon} f_{\varepsilon}^{\prime}\left(u_{\varepsilon r}\right) w_{r}$ in $\Omega_{\varepsilon} \times(0, \infty), f\left(u_{\varepsilon r}\right)$ is bounded in $\Omega_{\varepsilon} \times(0, \infty)$ due to boundedness of $f_{\varepsilon}$, and so is $u_{\varepsilon} f_{\varepsilon}^{\prime}\left(u_{\varepsilon r}\right)$ because of Lemma 20, we can apply [4, Prop. 52.8] to conclude nonpositivity of $w$ from nonpositivity of $w$ on $\Omega_{\varepsilon} \times\{0\}$ (see (4.24b)) and on $\partial \Omega_{\varepsilon} \times(0, \infty)$ as guaranteed by Lemmata 21 and 22.

We now turn our attention to the counterpart of Lemma 22.

Lemma 24. For every $\varepsilon>0$ we obtain

$$
u_{\varepsilon r}(\varepsilon, t) \geq-c_{\varepsilon}^{\star}
$$

for every $t \in(0, \infty)$, where $c_{\varepsilon}^{\star}$ is as in Definition 16.

Proof. We define $\underline{u}(r, t):=\left(u^{\star}-v\right)(\varepsilon, t)+c_{\varepsilon}^{\star}(\varepsilon-r)$. Then $\underline{u}(\varepsilon, t)=u_{\varepsilon}(\varepsilon, t)$ for all $t>0$ due to the boundary condition in (4.26); by (4.24a) and (4.25c),

$$
\underline{u}(r, 0)=u_{0 \varepsilon}(\varepsilon)-c_{\varepsilon}^{*}(r-\varepsilon) \leq u_{0 \varepsilon}(\varepsilon)-\int_{\varepsilon}^{r} \sup \left|u_{0 \varepsilon r}\right| \leq u_{0 \varepsilon}(r),
$$

for every $r \in(\varepsilon, R)$, and similarly by (4.25a),

$$
\underline{u}(R, t)=u^{\star}(\varepsilon)-v(\varepsilon, t)-c_{\varepsilon}^{\star}(R-\varepsilon) \leq u^{*}(\varepsilon)-c_{\varepsilon}^{\star}(R-\varepsilon) \leq u^{\star}(R)=u_{\varepsilon}(R, t)
$$

for every $t>0$. Due to Definition $17, f_{\varepsilon}\left(c_{\varepsilon}^{\star}\right)=\left(c_{\varepsilon}^{\star}\right)^{3}$ and hence, by Lemma 20 and (4.25d),

$$
\underline{u}_{t}-\Delta \underline{u}-u_{\varepsilon} f_{\varepsilon}\left(\underline{u}_{r}\right)=-v_{t}(\varepsilon, t)+\frac{n-1}{r} c_{\varepsilon}^{\star}+\left(c_{\varepsilon}^{*}\right)^{3} u_{\varepsilon} \leq e^{-\lambda^{2} t} c_{v}+\frac{n-1}{\varepsilon} c_{\varepsilon}^{*}+u^{\star}(\varepsilon)\left(c_{\varepsilon}^{*}\right)^{3} \leq 0 .
$$

Therefore, comparison ([4, Prop. 52.6]) implies

$$
u_{\varepsilon}(r, t) \geq \underline{u}(r, t) \quad \text { for all } t>0, r \in(\varepsilon, R),
$$

and as $u_{\varepsilon}(\varepsilon, t)=\underline{u}(\varepsilon, t)$ for every $t>0$, this shows that $u_{\varepsilon r}(\varepsilon, t) \geq \underline{u}_{r}(\varepsilon, t)=-c_{\varepsilon}^{\star}$ for every $t>0$.

The previous lemmata and a first Bernstein-type comparison of $u_{\varepsilon r}^{2}$ confirm that including $f_{\varepsilon}$ in (4.26) - although necessary for application of the classical existence theorems - has not altered the equation.

Lemma 25. For every $\varepsilon>0$ we have

$$
\sup _{\Omega_{\varepsilon} \times(0, \infty)}\left|\nabla u_{\varepsilon}\right| \leq c_{\varepsilon}^{\star}
$$

Proof. We let $\mathcal{M}[\phi]:=\phi_{t}-\Delta \phi-f_{\varepsilon}^{\prime}\left(u_{\varepsilon r}\right) u_{\varepsilon} \phi_{r}$. Then $\mathcal{M}\left[c_{\varepsilon}^{\star}\right]=0$ and

$$
\begin{aligned}
\mathcal{M}\left[\left|\nabla u_{\varepsilon}\right|^{2}\right]= & 2 \nabla u_{\varepsilon} \cdot \nabla \Delta u_{\varepsilon}+2\left|\nabla u_{\varepsilon}\right|^{2} f_{\varepsilon}\left(u_{\varepsilon r}\right)+2 u_{\varepsilon} f_{\varepsilon}^{\prime}\left(u_{\varepsilon r}\right) \nabla u_{\varepsilon} \cdot \nabla u_{\varepsilon r} \\
& -\nabla \cdot\left(2 D^{2} u_{\varepsilon} \nabla u_{\varepsilon}\right)-2 f_{\varepsilon}^{\prime}\left(u_{\varepsilon r}\right) u_{\varepsilon} \nabla u_{\varepsilon} \cdot \nabla u_{\varepsilon r} \\
= & 2\left|\nabla u_{\varepsilon}\right|^{2} f_{\varepsilon}\left(u_{\varepsilon r}\right)-2\left|D^{2} u_{\varepsilon}\right|^{2} \quad \text { in } \Omega_{\varepsilon} \times(0, \infty) .
\end{aligned}
$$

In view of Lemma 23, $\mathcal{N}\left[\left|\nabla u_{\varepsilon}\right|^{2}\right] \leq 0$. Lemma 21 and (4.25a) together with Lemmata 22 and 24 show that $\left(c_{\varepsilon}^{\star}\right)^{2} \geq\left|\nabla u_{\varepsilon}\right|^{2}$ on $\partial \Omega_{\varepsilon} \times(0, \infty)$, and (4.25c) ensures the same on $\Omega_{\varepsilon} \times\{0\}$. Therefore, comparison (in the form of [4, Prop. 52.10], if one allows $f$ to also depend on $t$ there - the necessary adaptations in the corresponding proof are minor) proves $\sup _{\Omega_{\varepsilon} \times(0, \infty)}\left|\nabla u_{\varepsilon}\right|^{2} \leq\left(c_{\varepsilon}^{\star}\right)^{2}$ and thus the lemma.

Lemma 26. The function $u_{\varepsilon}$ solves

$$
\begin{cases}u_{\varepsilon t}=\Delta u_{\varepsilon}+u_{\varepsilon} u_{\varepsilon r}^{3} & \text { in } \Omega_{\varepsilon} \times(0, \infty), \\ \left.u_{\varepsilon}\right|_{\partial B_{\varepsilon}}(\cdot, t)=u^{\star}-\left.v(\cdot, t)\right|_{\partial B_{\varepsilon}} & \text { for all } t>0, \\ \left.u_{\varepsilon}\right|_{\partial B_{R}}(\cdot, t)=u_{0}(R)=u^{\star}(R) & \text { for all } t>0, \\ u_{\varepsilon}(\cdot, 0)=u_{0 \varepsilon} & \text { in } \overline{\Omega_{\varepsilon}} .\end{cases}
$$


Proof. Lemma 25 guarantees that $\left|u_{\varepsilon r}\right|=\left|\nabla u_{\varepsilon}\right| \leq c_{\varepsilon}^{\star}$ in $\Omega_{\varepsilon} \times(0, \infty)$, therefore $f\left(u_{\varepsilon r}\right)=u_{\varepsilon r}^{3}$ by Definition 17, and Lemma 26 becomes a corollary of Lemma 18.

\subsection{A priori estimates}

Inspired by the reasoning in [2, Sec. 2], which goes back to [23], we will now obtain an $\varepsilon$-independent bound for $u_{\varepsilon r}$ from a comparison principle applied to, essentially, a large, even power of $u_{\varepsilon r}$. Lack of $\varepsilon$-independent control over $u_{\varepsilon r}$ on the inner boundary (for which we refer to Lemma 24 and which is natural if seen in light of the unbounded derivative of $u^{\star}$ near $r=0$ ) makes inclusion of a cutoff function necessary.

Lemma 27. Let $p \geq 4$ be an even integer. There is $c>0$ such that

$$
(r-\delta)_{+}^{p+3} u_{\varepsilon r}^{p}(r, t) \leq c\left(1+\sup _{r>\delta}(r-\delta)_{+}^{p+3} u_{0 \varepsilon r}^{p}+t\right)
$$

for every $\delta>0, \varepsilon \in(0, \delta)$ and $t>0, r \in(0, R)$.

Proof. We define $c:=\max \left\{1, R^{p+3}\left|u_{r}^{\star}(R)\right|^{p},(3(p+3))^{p+3}\left|u^{\star}(R)\right|^{p+3}+\left(R \frac{p(p+3)^{2}}{p-1}\right)^{\frac{p+3}{3}}\right\}$ and fix $\delta>0$ and $\varepsilon \in$ $(0, \delta)$. Letting $w(r, t):=(r-\delta)_{+}^{p+3} u_{\varepsilon r}^{p}(r, t)$ for $(r, t) \in(\delta, R) \times(0, \infty)$, in $(\delta, R) \times(0, \infty)$ we compute

$$
w_{r}=(p+3)(r-\delta)_{+}^{p+2} u_{\varepsilon r}^{p}+p(r-\delta)_{+}^{p+3} u_{\varepsilon r}^{p-1} u_{\varepsilon r r}
$$

and

$$
\begin{gathered}
w_{r r}=(p+2)(p+3)(r-\delta)_{+}^{p+1} u_{\varepsilon r}^{p}+2 p(p+3)(r-\delta)_{+}^{p+2} u_{\varepsilon r}^{p-1} u_{\varepsilon r r} \\
+p(p-1)(r-\delta)_{+}^{p+3} u_{\varepsilon r}^{p-2} u_{\varepsilon r r}^{2}+p(r-\delta)_{+}^{p+3} u_{\varepsilon r}^{p-1} u_{\varepsilon r r r}
\end{gathered}
$$

as well as

$$
u_{\varepsilon r t}=u_{\varepsilon r r}+\frac{n-1}{r} u_{\varepsilon r r}-\frac{n-1}{r^{2}} u_{\varepsilon r}+u_{\varepsilon r}^{4}+3 u_{\varepsilon} u_{\varepsilon r}^{2} u_{\varepsilon r r} .
$$

For $\mathcal{M}[\phi]:=\phi_{t}-\Delta \phi-3 u_{\varepsilon} u_{\varepsilon r}^{2} \phi_{r}$ we thus obtain from (4.28)

$$
\begin{aligned}
\mathcal{M}[w]= & p(r-\delta)_{+}^{p+3} u_{\varepsilon r}^{p-1} u_{\varepsilon r t}-w_{r r}-\frac{n-1}{r} w_{r}-3 u_{\varepsilon} u_{\varepsilon r}^{2} w_{r} \\
= & p(r-\delta)_{+}^{p+3} u_{\varepsilon r}^{p-1} u_{\varepsilon r r r}+p \frac{n-1}{r}(r-\delta)_{+}^{p+3} u_{\varepsilon r}^{p-1} u_{\varepsilon r r} \\
& -p \frac{n-1}{r^{2}}(r-\delta)_{+}^{p+3} u_{\varepsilon r}^{p}+p(r-\delta)_{+}^{p+3} u_{\varepsilon r}^{p+3}+3 p(r-\delta)_{+}^{p+3} u_{\varepsilon} u_{\varepsilon r}^{p+1} u_{\varepsilon r r} \\
& -(p+2)(p+3)(r-\delta)_{+}^{p+1} u_{\varepsilon r}^{p}-2 p(p+3)(r-\delta)_{+}^{p+2} u_{\varepsilon r}^{p-1} u_{\varepsilon r r} \\
& -p(p-1)(r-\delta)_{+}^{p+3} u_{\varepsilon r}^{p-2} u_{\varepsilon r r}^{2}-p(r-\delta)_{+}^{p+3} u_{\varepsilon r}^{p-1} u_{\varepsilon r r r} \\
& -(p+3) \frac{n-1}{r}(r-\delta)_{+}^{p+2} u_{\varepsilon r}^{p}-p \frac{n-1}{r}(r-\delta)_{+}^{p+3} u_{\varepsilon r}^{p-1} u_{\varepsilon r r} \\
& -3(p+3)(r-\delta)_{+}^{p+2} u_{\varepsilon} u_{\varepsilon r}^{p+2}-3 p(r-\delta)_{+}^{p+3} u_{\varepsilon} u_{\varepsilon r}^{p+1} u_{\varepsilon r r} \\
= & -p \frac{n-1}{r 2}(r-\delta)_{+}^{p+3} u_{\varepsilon r}^{p}+p(r-\delta)_{+}^{p+3} u_{\varepsilon r}^{p+3} \\
& -(p+3)(p+2)(r-\delta)_{+}^{p+1} u_{\varepsilon r}^{p}-2 p(p+3)(r-\delta)_{+}^{p+2} u_{\varepsilon r}^{p-1} u_{\varepsilon r r} \\
& -p(p-1)(r-\delta)_{+}^{p+3} u_{\varepsilon r}^{p-2} u_{\varepsilon r r}^{2} \quad \text { in }\left(B_{R} \backslash B_{\delta}\right) \times(0, \infty) . \\
& -(p+3) \frac{n-1}{r}(r-\delta)_{+}^{p+2} u_{\varepsilon r}^{p}-3(p+3)(r-\delta)_{+}^{p+2} u_{\varepsilon} u_{\varepsilon r}^{p+2} \\
\leq & p(r-\delta)_{+}^{p+3} u_{\varepsilon r}^{p+3}-2 p(p+3)(r-\delta)_{+}^{p+2} u_{\varepsilon r}^{p-1} u_{\varepsilon r r}-p(p-1)(r-\delta)_{+}^{p+3} u_{\varepsilon r}^{p-2} u_{\varepsilon r r}^{2} \\
& -3(p+3)(r-\delta)_{+}^{p+2} u_{\varepsilon} u_{\varepsilon r}^{p+2} \quad
\end{aligned}
$$


Here, by Young's inequality

$$
\begin{aligned}
& -2 p(p+3)(r-\delta)_{+}^{p+2} u_{\varepsilon r}^{p-1} u_{\varepsilon r r} \\
& \leq p(p-1)(r-\delta)_{+}^{p+3} u_{\varepsilon r}^{p-2} u_{\varepsilon r r}^{2}+\frac{p(p+3)^{2}}{p-1}(r-\delta)^{p+1} u_{\varepsilon r}^{p} \\
& \leq p(p-1)(r-\delta)_{+}^{p+3} u_{\varepsilon r}^{p-2} u_{\varepsilon r r}^{2}+(r-\delta)_{+}^{p+3}\left|u_{\varepsilon r}\right|^{p+3}+\left((r-\delta)_{+} \frac{p(p+3)^{2}}{p-1}\right)^{\frac{p+3}{3}}
\end{aligned}
$$

and

$$
-3(p+3)(r-\delta)_{+}^{p+2} u_{\varepsilon} u_{\varepsilon r}^{p+2} \leq(r-\delta)_{+}^{p+3}\left|u_{\varepsilon r}\right|^{p+3}+(3(p+3))^{p+3}\left|u_{\varepsilon}\right|^{p+3}
$$

in $\left(B_{R} \backslash B_{\delta}\right) \times(0, \infty)$. Recalling the sign of $u_{\varepsilon r}$ from Lemma 23 and setting $c_{1}:=(3(p+3))^{p+3}\left|u^{\star}(R)\right|^{p+3}+$ $\left(R \frac{p(p+3)^{2}}{p-1}\right)^{\frac{p+3}{3}}$ we hence obtain

$$
\mathcal{M}[w] \leq c_{1} \quad \text { in }\left(B_{R} \backslash B_{\delta}\right) \times(0, \infty) .
$$

Furthermore,

$$
w(R, t) \leq R^{p+3} u_{\varepsilon r}^{p}(R, t) \leq R^{p+3}\left(u_{r}^{\star}(R)\right)^{p}=: c_{2} \quad \text { for all } t>0
$$

by Lemma 21. With $c=\max \left\{c_{1}, c_{2}, 1\right\}$ and $\bar{w}:=c\left(1+\sup _{r>\delta}(r-\delta)_{+}^{p+3} u_{0 \varepsilon r}^{p}+t\right)$ we not only have $\mathcal{M}[\bar{w}]=c \geq$ $\mathcal{M}[w]$ in $\left(B_{R} \backslash B_{\delta}\right) \times(0, \infty)$, but also $\bar{w}(R, t) \geq c_{2} \geq w(R, t)$ for all $t>0$ and $\bar{w}(r, 0) \geq \sup _{r>\delta}(r-\delta)_{+}^{p+3} u_{0 \varepsilon r}^{p} \geq w(r, 0)$ for all $r \in(0, R)$ as well as $\bar{w}(\delta, t) \geq 0=w(\delta, t)$ for all $t>0$. Comparison (again by means of an adaptation of [4, Prop. 52.10]) allows us to conclude $(r-\delta)_{+}^{p+3} u_{\varepsilon r}^{p}=w \leq \bar{w}=c\left(1+\sup _{r>\delta}(r-\delta)_{+}^{p+3} u_{0 \varepsilon r}^{p}+t\right) \operatorname{in}\left(B_{R} \backslash B_{\delta}\right) \times(0, \infty)$. Additionally, for $r \in(0, \delta)$, the left-hand side of this inequality is zero, and (4.29) holds.

Next we bring Lemma 27 in a more directly applicable form.

Lemma 28. Let $p \geq 4$ be an even integer. For every $T>0$ there is $c>0$ such that

$$
\left|u_{\varepsilon r}(r, t)\right| \leq c r^{-\frac{p+3}{p}}
$$

for every $\varepsilon>0, t \in[0, T], r \in(2 \varepsilon, R)$.

Proof. Conditions (2.14f) and (4.24b) ensure the existence of $c_{1}>0$ such that

$$
\left|u_{0 \varepsilon r}\right| \leq c_{1} r^{-\frac{2}{3}} \text { on } B_{R} \backslash B_{\varepsilon}
$$

for every $\varepsilon>0$, and hence

$$
(r-\delta)_{+}^{p+3} u_{0 \varepsilon r}^{p} \leq c_{1}(r-\delta)_{+}^{p+3} r^{-\frac{2 p}{3}}
$$

for every $r \in(\delta, R)$ and $\varepsilon<\delta$. Noting that $r \mapsto(r-\delta)_{+}^{p+3} r^{-\frac{2 p}{3}}$ is increasing on $(\delta, R)$ due to $p+3>\frac{2 p}{3}$, we conclude that

$$
(r-\delta)_{+}^{p+3} u_{0 \varepsilon r}^{p} \leq c_{1} R^{p-\frac{2 p}{3}}=c_{1} R^{\frac{p}{3}}
$$

for every $r \in(\delta, R)$ and $\varepsilon \in(0, \delta)$. Lemma 27 hence implies that there is $c_{2}>0$ such that

$$
(r-\delta)_{+}^{p+3} u_{\varepsilon r}^{p}(r, t) \leq c_{2}(1+t)
$$

for every $\delta>0, \varepsilon \in(0, \delta)$ and $t>0, r \in(0, R)$. If we insert $r=2 \delta$, we obtain

$$
\left|u_{\varepsilon r}(2 \delta, t)\right| \leq c_{3}(2 \delta)^{-\frac{p+3}{p}}(1+t)
$$

for every $\delta>0, \varepsilon \in(0, \delta), t>0$, where $c_{3}:=2^{\frac{p+3}{p}} c_{2}$. We conclude by letting $c:=c_{3}(1+T)$.

As preparation of the compactness argument that will finally establish existence of a solution of $(0.5)$ in $\left(B_{R} \backslash\right.$ $\{0\}) \times(0, \infty)$, we use classical regularity theory for parabolic PDEs and rely on Lemma 28 as a starting point. 
Lemma 29. Let $\beta \in(0,1)$. Let $K$ be a compact subset of $\left(B_{R} \backslash\{0\}\right) \times(0, \infty)$. Then there are $\varepsilon_{0}>0$ and $c>0$ such that for every $\varepsilon \in\left(0, \varepsilon_{0}\right)$

$$
\left\|u_{\varepsilon}\right\|_{C^{1+\beta, \frac{1+\beta}{2}}(K)} \leq c .
$$

Proof. Let us choose $\delta>0$ so small that $\left(B_{\delta} \times(0, \infty)\right) \cap K=\emptyset$. Let $\eta \in C_{c}^{\infty}\left(\left(B_{R} \backslash B_{\delta}\right) \times(0, \infty)\right)$ be such that $\eta \equiv 1$ on $K$. Then for each $\varepsilon \in\left(0, \varepsilon_{0}\right), \varepsilon_{0}:=\frac{\delta}{2}, \eta u_{\varepsilon}$ is well-defined on $\left(B_{R} \backslash B_{\delta}\right) \times(0, \infty)$ and $\left(\eta u_{\varepsilon}\right)(\delta, t)=0$, $\left(\eta u_{\varepsilon}\right)(R, t)=0$ for every $t>0,\left(\eta u_{\varepsilon}\right)(r, 0)=0$ for every $r \in(\delta, R)$ and

$$
\left(\eta u_{\varepsilon}\right)_{t}=\Delta\left(\eta u_{\varepsilon}\right)+g_{\varepsilon} \quad \text { in }\left(B_{R} \backslash B_{\delta}\right) \times(0, \infty),
$$

where $g_{\varepsilon}:=-u_{\varepsilon} \Delta \eta-2 \nabla u_{\varepsilon} \cdot \nabla \eta+\eta u_{\varepsilon} u_{\varepsilon r}^{3}-\eta_{t} u_{\varepsilon}$. Lemma 28 enables us to find $c_{1}>0$ satisfying

$$
\left\|g_{\varepsilon}\right\|_{L^{\infty}\left(\left(B_{R} \backslash B_{\delta}\right) \times(0, \infty)\right)}=\left\|g_{\varepsilon}\right\|_{L^{\infty}(\operatorname{supp} \eta)} \leq c_{1}
$$

for every $\varepsilon \in\left(0, \varepsilon_{0}\right)$. Consequently, [26, Thm. 7.4, p. 191] shows that with some $c_{2}>0$,

$$
\left\|\eta u_{\varepsilon}\right\|_{C^{1+\beta, \frac{1+\beta}{2}}\left(\left(B_{R} \mid B_{\delta}\right) \times(0, \infty)\right)} \leq C_{2} \quad \text { for every } \varepsilon \in\left(0, \varepsilon_{0}\right) .
$$

Leveraging Lemma 29, we can achieve higher regularity analogously.

Lemma 30. Let $\beta \in(0,1)$. Let $K$ be a compact subset of $\left(B_{R} \backslash\{0\}\right) \times(0, \infty)$. Then there are $\varepsilon_{0}>0$ and $c>0$ such that

$$
\left\|u_{\varepsilon}\right\|_{C^{2+\beta, 1+\frac{\beta}{2}(K)}} \leq c \quad \text { for every } \varepsilon \in\left(0, \varepsilon_{0}\right) .
$$

Proof. Again, we choose $\delta>0$ so small that $\left(B_{\delta} \times(0, \infty)\right) \cap K=\emptyset, \eta \in C_{c}^{\infty}\left(\left(B_{R} \backslash B_{\delta}\right) \times(0, \infty)\right)$ such that $\eta \equiv 1$ on $K$ and $\varepsilon_{0}:=\delta$ and consider the Dirichlet problem of $\left(\eta u_{\varepsilon}\right)_{t}=\Delta\left(\eta u_{\varepsilon}\right)+g_{\varepsilon}$ in $\left(B_{R} \backslash B_{\delta}\right) \times(0, \infty)$, with $g_{\varepsilon}:=-u_{\varepsilon} \Delta \eta-2 \nabla u_{\varepsilon} \cdot \nabla \eta+\eta u_{\varepsilon} u_{\varepsilon r}^{3}-\eta_{t} u_{\varepsilon}$. Thanks to Lemma 29, applied to the compact set supp $\eta$, there is $c_{1}>0$ fulfilling

$$
\left\|g_{\varepsilon}\right\|_{C^{\beta, \frac{\beta}{2}}\left(\left(B_{R} \backslash B_{\delta}\right) \times(0, \infty)\right)}=\left\|g_{\varepsilon}\right\|_{C^{\beta, \frac{\beta}{2}}(\operatorname{supp} \eta)} \leq c_{1} \quad \text { for every } \varepsilon \in\left(0, \varepsilon_{0}\right) .
$$

We can therefore rely on [26, Thm. 3.6, p. 65] so as to conclude the existence of $c_{2}>0$ such that

$$
\left\|\eta u_{\varepsilon}\right\|_{C^{2+\beta, 1+\frac{\beta}{2}}\left(\left(B_{R} \backslash B_{\delta}\right) \times(0, \infty)\right)} \leq c_{2} \quad \text { for every } \varepsilon \in\left(0, \varepsilon_{0}\right) .
$$

In the next step we aim for lower Hölder regularity, but strive to include the boundaries at $r=R$ and $t=0$.

Lemma 31. There is $\beta \in(0,1)$ such that for every compact subset $K$ of $\left(\bar{B}_{R} \backslash\{0\}\right) \times[0, \infty)$ there are $\varepsilon_{0}>0$ and $c>0$ satisfying

$$
\left\|u_{\varepsilon}\right\|_{C^{\beta, \frac{\beta}{2}(K)}} \leq c \quad \text { for every } \varepsilon \in\left(0, \varepsilon_{0}\right) .
$$

Proof. We choose $\delta>0$ so small that $\left(B_{\delta} \times(0, \infty)\right) \cap K=\emptyset$ and let $\varepsilon_{0} \in\left(0, \frac{\delta}{2}\right)$ be such that $u_{0 \varepsilon}=u_{0}$ on $B_{R} \backslash B_{\delta}$ for every $\varepsilon \in\left(0, \varepsilon_{0}\right)$ (cf. Remark 15). With $\eta \in C_{c}^{\infty}\left(\left(B_{R} \backslash B_{\delta}\right) \times(0, \infty)\right)$ such that $\eta \equiv 1$ on $K$ and relying on Lemma 28, we can conclude from [1, Thm. III.10.1] that with some $c>0$,

$$
\left\|\eta u_{\varepsilon}\right\|_{C^{\beta, \frac{\beta}{2}(K)}} \leq c \quad \text { for every } \varepsilon \in\left(0, \varepsilon_{0}\right),
$$

where $\beta$ can be determined independently of $\delta, K$ and $\eta$.

\subsection{Solving the limit problem}

With these estimates at hand, we are ready to carry out the existence proof. 
Lemma 32. There is a function $u \in C\left(\bar{B}_{R} \times[0, \infty)\right) \cap C^{2,1}\left(\left(B_{R} \backslash\{0\}\right) \times(0, \infty)\right)$ solving (0.5). This function is radially symmetric, satisfies

$$
u^{\star}(r) \geq u(r, t) \geq u^{\star}(r)-v(r, t) \quad \text { for all }(r, t) \in[0, R] \times[0, \infty)
$$

and, in particular, with some $c>0$ we have

$$
0 \geq u \geq-c r^{\frac{1}{3}} \quad \text { in } B_{R} \times[0, \infty),
$$

as well as

$$
u_{r} \leq 0 \quad \text { in }\left(B_{R} \backslash\{0\}\right) \times(0, \infty),
$$

and for every $T>0$ there is some $c=c(T)>0$ such that

$$
u_{r}>-c r^{-\frac{31}{28}} \quad \text { in }\left(B_{R} \backslash\{0\}\right) \times(0, T) .
$$

Proof. If we apply Lemmata 30 and 31 to sequences of compact sets exhausting $\left(B_{R} \backslash\{0\}\right) \times(0, \infty)$ and $\left(\bar{B}_{R} \backslash\{0\}\right) \times[0, \infty)$, respectively, use the Arzelà-Ascoli theorem and a diagonalization procedure, we obtain a sequence $\left(\varepsilon_{j}\right)_{j \in \mathbb{N}} \vee 0$ and a function $u \in C\left(\left(\bar{B}_{R} \backslash\{0\}\right) \times[0, \infty)\right) \cap C^{2,1}\left(\left(B_{R} \backslash\{0\}\right) \times(0, \infty)\right)$ such that

$$
\begin{aligned}
u_{\varepsilon_{j}} \rightarrow u & \text { locally uniformly in }\left(\bar{B}_{R} \backslash\{0\}\right) \times[0, \infty) \\
& \text { and with respect to the topology of } C^{2,1}\left(\left(B_{R} \backslash\{0\}\right) \times(0, \infty)\right) .
\end{aligned}
$$

The latter convergence statement (4.35) together with Lemma 23 already entails (4.32), whereas (4.33) similarly results from Lemma 28 upon the choice of $p=28$.

Additionally, we define $u(0, t):=0$. Then $u$ is continuous in $\bar{B}_{R} \times[0, \infty)$. In light of (4.34), only continuity at $(0, t)$ for $t \geq 0$ remains to be proven. Let $\eta>0$. Choose $\delta>0$ such that $u^{\star}(\delta)-v(\delta, 0)>-\eta$. Then for every $\varepsilon \in(0, \delta)$, every $r \in(0, \delta)$ and every $t \geq 0$ we have $0 \geq u_{\varepsilon}(r, t) \geq u_{\varepsilon}(\delta, t) \geq u^{*}(\delta)-v(\delta, t) \geq u^{*}(\delta)-v(\delta, 0)>-\eta$ and, by (4.34), hence $0 \geq u(r, t) \geq-\eta$ for every $r \in(0, \delta)$ and $t \geq 0$.

Finally, (4.30) and hence (4.31) are obvious for $r=0$ and easily obtained from Lemma 20 for $r>0$.

Theorem 1 also includes a uniqueness statement. The following lemma takes care of it.

Lemma 33. Let $u, \tilde{u}$ be functions satisfying

$$
\begin{aligned}
& u, \tilde{u} \in C^{2,1}\left(\left(B_{R} \backslash\{0\}\right) \times(0, \infty)\right) \cap C\left(\bar{B}_{R} \times[0, \infty)\right), \\
& \sup u_{r}<\infty, \quad \sup \tilde{u}_{r}<\infty
\end{aligned}
$$

that solve (0.5). (The suprema are supposed to be taken over $\left(B_{R} \backslash\{0\}\right) \times(0, \infty)$.) Then $u=\tilde{u}$.

Proof. The difference $w:=u-\tilde{u}$ solves $w_{t}=\Delta w+b w_{r}+c w$ in $\left.\left(B_{R} \backslash\{0\}\right) \times(0, \infty)\right)$, where $b:=\tilde{u}\left(u_{r}^{2}+u_{r} \tilde{u}_{r}+\tilde{u}_{r}^{2}\right)$ and $c:=u_{r}^{3}$ has a finite supremum. Moreover, $w=0$ on $\left(B_{R} \times\{0\}\right) \cup\left(\partial\left(B_{R} \backslash\{0\}\right) \times(0, \infty)\right)$, and [4, Prop. 52.4] shows $w \leq 0$.

The final piece of the proof of Theorem 2 is the combination of Lemma 32 with Lemma 4.

Lemma 34. Let $n \geq 3$. Then the function $u$ obtained in Lemma 32 is a weak solution of (0.7).

Proof. We observe that according to (4.33) there is $c_{1}=c_{1}(T)$ such that

$$
\frac{1}{\varepsilon} \int_{0}^{T} \int_{0}^{\varepsilon} r^{n-1}\left|u_{r}(r, t)\right| d r d t \leq \frac{c_{1} T}{\varepsilon} \int_{0}^{\varepsilon} r^{n-1} r^{-\frac{31}{28}} d r=\frac{c_{1} T}{n-\frac{31}{28}} \varepsilon^{n-\frac{59}{28}} \rightarrow 0
$$

as $\varepsilon \rightarrow 0$. By (4.33) and (4.31)

$$
\left|u u_{r}^{3}\right| \leq c r^{\frac{1}{3}} r^{3 \cdot\left(-\frac{31}{28}\right)}=c r^{\frac{28-9 \cdot 31}{84}}=c r^{-\frac{251}{84}} \quad \text { in } B_{R} \times(0, \infty),
$$

and because $-\frac{251}{84}=-3+\frac{1}{84} \geq-n$, hence $u u_{r}^{3} \in L_{l o c}^{1}\left(B_{R} \times(0, \infty)\right)$. Finally, $\left|u_{r}\right| \leq c r^{-\frac{31}{28}} \in L_{l o c}^{1}$ and Lemma 4 becomes applicable. 


\section{Proofs of the theorems}

Proof of Theorem 1 and Remark 7. Solvability is ensured by Lemma 32, which by means of (4.30) also ensures that for every $t>0$ there are $c_{1}=c_{1}(t)>0$ and $c_{2}=c_{2}(t)>0$ such that

$$
0 \geq u^{*}(r)-u(r, t) \geq-v(r, t) \geq-c_{1} r^{n-\frac{3}{2}} J_{v}(\lambda r) \geq-c_{2} r^{n-\frac{3}{2}+v} \quad \text { for every } r \in[0, R] .
$$

(The last estimate therein used $\lambda R<x_{0}$ and [24, p. 360, (9.1.7)].) This proves Remark 7 and implies (0.6). Uniqueness of solutions, on the other hand, has been asserted in Lemma 33.

Proof of Theorem 2. This is the outcome of Lemma 34.

Proof of Theorem 3. The construction of $u$ during the proof of Theorem 1 had ensured that $u^{\star}(r) \geq u(r, t) \geq$ $u^{\star}(r)-v(r, t)$ for all $(r, t) \in[0, R] \times[0, \infty)$ (cf. (4.30)), and Theorem 3 can be seen from the explicit definition (3.15) of $v$.

Acknowledgements: The first author was supported in part by the Slovak Research and Development Agency under the contract No. APVV-18-038 and by the VEGA grant 1/0347/18.

\section{References}

[1] O. A. Ladyženskaja, V. A. Solonnikov and N. N. Ural'ceva, Linear and quasilinear equations of parabolic type, Translated from the Russian by S. Smith. Translations of Mathematical Monographs, Vol. 23, American Mathematical Society, Providence, R.I. (1968).

[2] S. B. Angenent and M. Fila, Interior gradient blow-up in a semilinear parabolic equation., Differ. Integral Equ., 9 (1996), no. 5, 865-877.

[3] M. Fila and J. Lankeit, Continuation beyond interior gradient blow-up in a semilinear parabolic equation, Mathematische Annalen, (2019), 10.1007/s00208-019-01827-2.

[4] P. Quittner and Ph. Souplet, Superlinear parabolic problems. Blow-up, global existence and steady states., Basel: Birkhäuser (2007).

[5] E. Chasseigne, Classification of razor blades to the filtration equation - the sublinear case., J. Differ. Equations, 187 (2003), no. 1, 72-105.

[6] E. Chasseigne and J. L. Vazquez, Theory of extended solutions for fast-diffusion equations in optimal classes of data. Radiation from singularities., Arch. Ration. Mech. Anal., 164 (2002), no. 2, 133-187.

[7] E. Chasseigne and J. L. Vázquez, The pressure equation in the fast diffusion range., Rev. Mat. Iberoam., 19 (2003), no. 3, 873-917.

[8] K. M. Hui and S. Kim, Asymptotic large time behavior of singular solutions of the fast diffusion equation., Discrete Contin. Dyn. Syst., 37 (2017), no. 11, 5943-5977.

[9] F. Quirós and J. L. Vázquez, Asymptotic behaviour of the porous media equation in an exterior domain., Ann. Sc. Norm. Super. Pisa, Cl. Sci., IV. Ser., 28 (1999), no. 2, 183-227.

[10] J. L. Vázquez and M. Winkler, The evolution of singularities in fast diffusion equations: infinite-time blow-down., SIAM J. Math. Anal., 43 (2011), no. 4, 1499-1535.

[11] M. Fila, J. Takahashi and E. Yanagida, Solutions with moving singularities for equations of porous medium type., Nonlinear Anal., Theory Methods Appl., 179 (2019), 237-253.

[12] T. Kan and J. Takahashi, On the profile of solutions with time-dependent singularities for the heat equation., Kodai Math. J., 37 (2014), no. 3, 568-585.

[13] J. Takahashi and E. Yanagida, Time-dependent singularities in the heat equation., Commun. Pure Appl. Anal., 14 (2015), no. 3, 969-979.

[14] T. Kan and J. Takahashi, Time-dependent singularities in semilinear parabolic equations: behavior at the singularities., J. Differ. Equations, 260 (2016), no. 10, 7278-7319.

[15] T. Kan and J. Takahashi, Time-dependent singularities in semilinear parabolic equations: existence of solutions., J. Differ. Equations, 263 (2017), no. 10, 6384-6426.

[16] S. Sato and E. Yanagida, Solutions with moving singularities for a semilinear parabolic equation., J. Differ. Equations, 246 (2009), no. 2, 724-748.

[17] S. Sato and E. Yanagida, Forward self-similar solution with a moving singularity for a semilinear parabolic equation., Discrete Contin. Dyn. Syst., 26 (2010), no. 1, 313-331. 
[18] S. Sato and E. Yanagida, Singular backward self-similar solutions of a semilinear parabolic equation., Discrete Contin. Dyn. Syst., Ser. S, 4 (2011), no. 4, 897-906.

[19] J. Takahashi and E. Yanagida, Time-dependent singularities in a semilinear parabolic equation with absorption., Commun. Contemp. Math., 18 (2016), no. 5, 27.

[20] M. Hoshino and E. Yanagida, Convergence rate to singular steady states in a semilinear parabolic equation., Nonlinear Anal., Theory Methods Appl., 131 (2016), 98-111.

[21] S. Sato and E. Yanagida, Asymptotic behavior of singular solutions for a semilinear parabolic equation., Discrete Contin. Dyn. Syst., 32 (2012), no. 11, 4027-4043.

[22] W.-M. Ni and P. Sacks, Singular behavior in nonlinear parabolic equations., Trans. Am. Math. Soc., 287 (1985), 657-671.

[23] S. Bernstein, Sur la généralisation du problème de Dirichlet. Deuxième partie., Math. Ann., 69 (1910), 82-136.

[24] Handbook of mathematical functions with formulas, graphs, and mathematical tables. 10th printing, with corrections., National Bureau of Standards. New York etc.: John Wiley \& Sons. (1972).

[25] G. M. Lieberman, The first initial-boundary value problem for quasilinear second order parabolic equations., Ann. Sc. Norm. Super. Pisa, Cl. Sci., IV. Ser., 13 (1986), 347-387.

[26] A. Friedman, Partial differential equations of parabolic type., Englewood Cliffs, N.J.: Prentice-Hall, Inc. XIV (1964). 